\title{
The Brain's Sequential Parallelism: Perceptual Decision-Making and Early Sensory Responses
}

\author{
Tobias Brosch ${ }^{\star}$ and Heiko Neumann \\ Ulm University, Germany \\ \{tobias.brosch, heiko.neumann\}@uni-ulm.de
}

\begin{abstract}
Multi-stage decision tasks require the determination of intermediate results in order to perform consecutive decision steps. Electrophysiological recordings in sensory, parietal, and pre-frontal cortical areas have demonstrated that different response characteristics and timings at the neuron level provide key mechanisms to implement characteristic functionalities. We propose a hybrid neural model architecture that accounts for such findings and quantitatively reproduces the timing of such responses. We demonstrate by numerical simulations how the model accounts for feature-dependent decisions and how these are sequentialized during mutual interactions of pools of neurons in different cortical areas. Feedback from higher-level areas to early sensory stages of processing establishes a link between mechanisms involved in response integration and target selection to representations of sensory input.
\end{abstract}

Keywords: Multi-stage decision, Electrophysiological recordings, Hybrid neural model architecture.

\section{Introduction}

Human behavior consists of the execution of routine tasks such as visual navigation, listening to auditory information, or producing speech during social conversation. Complex behavioral activities, such as the preparation of a cup of tea, or lane changing during an attempt of overtaking a car, require a sequence of intermediate decisions depending on results of previous processes. While early processing stages concerned with primary sensory processing, such as vision, consist of massive parallel computational steps, physiological studies [1, 2] showed a serial bottleneck in multi-step decision tasks (requiring interim-results to initiate the next processing step).

Taken together, these findings provide evidence that the brain's processing is hybrid in its nature, suggesting a hierarchical arrangement of parallel processes some of which are scheduled in serial order. In turn, this provides evidence for the theory of visual routines proposed by [3]. The control of how different processes are scheduled in a correct order requires adjusted timings of their execution. The underlying processing steps, like the learning of serialization of processes in working memory tasks [4] or massively parallel processing in object recognition [5-7]

\footnotetext{
* Corresponding author.

T. Huang et al. (Eds.): ICONIP 2012, Part II, LNCS 7664, pp. 41-50 2012.

(C) Springer-Verlag Berlin Heidelberg 2012
} 
have already been investigated. However, so far only little research has been devoted to the interaction of parallel and serial processes and their concerted integrative action for sequential task analysis. Evidence that visual routines are implemented and scheduled neurally to execute sequential sub-tasks has been gathered by recent physiological investigations [2, 8]. Recently, a first implementation of interacting sequential/parallel processes has been shown in [9] as part of a larger framework proposed in [10]. We continue this line of investigations but focus on the definition of a modular architecture to realize sequentially initiated parallel processing in a feedforward-feedback (FF-FB) network model. A hybrid architecture is proposed that employs a serial production system relying on decisions found by the massively parallel processing of the FF-FB network. We show that the necessarily different response properties of neurons can be derived from a generic neurodynamical neuron model and its embedding into a cascade of processing stages. We suggest that the FF-FB network interactions are used to compute intermediate decision results in which early areas act as a neural blackboard [11]. A sequential control mechanism uses these results to manipulate higher network areas which initiates a new decision process. Early model areas contribute to the decision process through indirect feedback interaction.

The remainder of this paper is organized as follows: Section 2 develops a description of model neurons which describe the dynamics of sequential decisionmaking processes in sect. 3 Section 4 introduces the dynamics of single decision steps in cascaded decision tasks described in sect. 5. Finally, sect. 6] summarizes the results and outlines the postulations of our model.

\section{Model Neurons}

We consider a layered architecture in which each layer contains a number of model neurons which are arranged on a discretized grid. We use a single-compartment neuron model with conductance-based dynamics to define the membrane potential $y$ (extended from [12, 13]), which is rectified to prevent negative values. The neural firing rates are generated by passing the activities through a sigmoidal non-linearity $\Psi$. The activation dynamics of a neuron $n_{i}$ is defined by

$$
\begin{aligned}
\tau \cdot \frac{d}{d t} y= & -\beta y+\left(\alpha_{E}-y\right) \cdot \Psi_{E}\left(I^{E}\right) \cdot\left(1+\gamma \cdot \Psi_{M}\left(I^{M}\right)\right) \\
& -\left(\alpha_{I}+y\right) \cdot \Psi_{I}\left(I^{I}\right)-\gamma_{\text {pool }} \cdot y \cdot \Psi_{\text {pool }}\left(y_{\text {pool }}\right), \\
\tau_{p} \cdot \frac{d}{d t} y_{\text {pool }}= & -y_{\text {pool }}+\left(\alpha_{\text {pool }}-y_{\text {pool }}\right) \cdot\left\{y * \Lambda_{\text {pool }}\right\},
\end{aligned}
$$

where the excitatory, modulatory and inhibitory inputs $I^{E}, I^{M}$ and $I^{I}$ are calculated as the weighted sum of the activities of connected input neurons $n_{j}, \Psi_{E / M / I}$ are sigmoidal function 1 (see Fig. 1, left), * denotes the spatial convolution

\footnotetext{
${ }_{1} \Psi(x)=\mathcal{H}(x) \cdot\left(2 \cdot\left(1+\exp \left(-4 \cdot x^{2}\right)\right)^{-1}-1\right)(\mathcal{H}(x)$ is the Heaviside step function), $\Psi_{E}(x)=\Psi_{M}(x)=\Psi(x), \Psi_{I}(x)=\Psi(6 \cdot(x-0.3)), \Psi_{\text {pool }}(x)=\Psi(3 \cdot(x-0.1))$.
} 

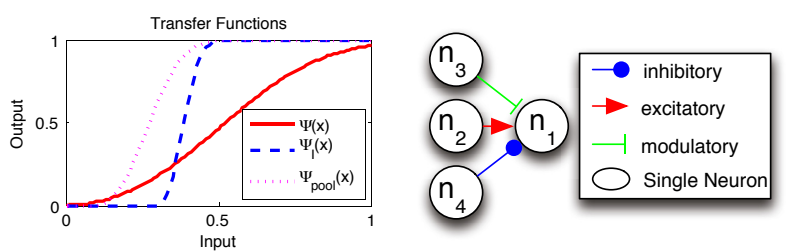

Fig. 1. Left: Sigmoidal transfer functions $\Psi$ to define the firing rates. Right: Symbols used to indicate connection types to a model neuron. Each of the single neurons is modeled by the activation dynamics of equation (1). Neuron $n_{1}$ receives excitatory/modulatory/inhibitory input from neurons $n_{2 / 3 / 4}$.

operator and $\Lambda^{\text {pool }}$ is a Gaussian low-pass filter (with standard deviation $\sigma_{\text {pool }}$ ) 2 . The symbols used in illustrations are shown in Fig. 1(right). Neurons at different stages in the brain differ in their response behaviors, ranging from transient and tonic sensory to integrating (or ramping) and sustained response characteristics. Different variants of the generic model introduced above can now be derived to account for the different response behaviors.

Self-Excitation: Sustained neuronal activity is modeled using self-exciting neurons. Once these self-excited neurons get enough driving input, they reverberate their activity remaining active for a while. Strong inhibition can deactivate them.

Tracing Operator: Contour tracing starting at some initially active spot in a $2 \mathrm{D}$ grid of neurons can be achieved using the tracing operator (compare [8]). Only neurons being excited and having active neurons in their neighborhood can be activated. The dynamics are those of equation (11) with the term $\Psi_{E}\left(I^{E}\right)$ replaced by $\Psi_{E}\left(I^{E} \cdot\left[\left\{y * \Lambda_{\text {trace }}\right\}-\varepsilon\right]_{+}\right), \Lambda_{\text {trace }}$ denoting a Gaussian filter.

Sensory, Ramping and Working Memory Neurons: Minor changes of the dynamics of eq. (11) allow us to model different types of neurons:

- Sensory: The firing rate of eq. (11) rises when excited and drops to a resting level when excitation is released.

- Ramping: Self-exciting neurons that integrate activity until a threshold $(75 \%$ of maximal activity) is reached. Then activity is reset to resting level.

- Working memory: Self-exciting neurons with slow inhibition of self-excitation resemble neurons staying active for a prolonged period of time.

\section{Modeling the Dynamics of Decision-Making}

Many perceptual or cognitive tasks require a decision between multiple alternatives. Consider, for example, a choice task with two alternatives (2AFC) to

\footnotetext{
${ }^{2}$ The parameters are set as follows: $\tau=0.25, \tau_{p}=0.5, \alpha_{E}=1, \alpha_{I}=3, \beta=1, \gamma=$ $2, \alpha_{\text {pool }}=1, \gamma_{\text {pool }}=1, \sigma_{\text {pool }}=1.5$. This keeps $y$ and $y^{\text {pool }}$ in the range of $[0,1]$ and decreases activity to zero for strong inhibition.
} 
decide whether a moving dot pattern moves to the left or to the right. In order to arrive at such a decision, evidence for leftward or rightward motion in the sensory input stream must be accumulated over time until a reliability threshold is reached. Reaching the threshold level triggers an eye movement towards the corresponding target location and quickly resets the integrated activity [14, 15]. Figure 2 displays how competitive ramping model neurons can solve a 2AFC task. Mutual inhibition between the neurons representing the choices "Left" and "Right" results in one of them winning the competition. Self-excitation assures saturation of neuronal activity.
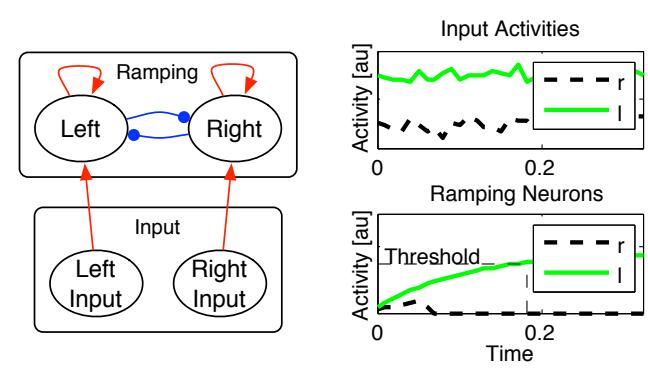

Fig. 2. Left: Neuronal Setup in the 2AFC task. Connections: excitatory (red arrows), inhibitory (bullet-head blue lines). Right: Simulation results for a 2AFC task of the network with dominating leftward motion input. The dominating input that is integrated by the associated ramping neuron results in a decision for the leftward direction.

\section{Modeling Individual Decision Steps}

Before we describe the full hybrid model, this section exemplarily demonstrates the way in which interim decisions are found in the FF-FB part of our model. The model architecture utilizes lower visual areas that serve as a blackboard for communication between higher cortical areas [11]. Here, we consider a search task in which a subject performs a saccade to one of two colored disks which has the same color as the initially fixated disk (sub-task used in [1, 2], see Fig. 3, left). This can be accomplished in cortex by selectively binding position information and feature properties like in the Where and What system [16]. In our model architecture we utilize a color feature representation which may be considered as a simplified version of the cortical ventral pathway. A global color category is incorporated as well. Spatial locations can be selected through an interaction between dorsal, parietal and prefrontal cortical representations, such as in area LIP and the frontal and supplemental eye fields (FEF, SEF). Functionally, an initially indexed spatial focus is generated and maintained in a spatial focus map to enhance features located at the fixation spot through temporal binding to a feature map "Color" (see Interaction with the general feature categories in "Global Color" results in a read out operation through competition between the individual feature categories. The winning feature category provides enhanced feedback to the feature map "Color" effectively performing a spatial selection. Fig. 3, right). 

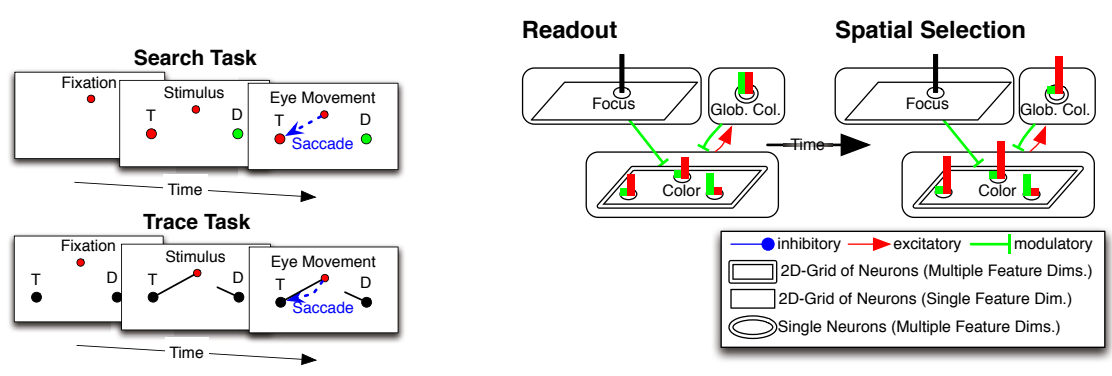

Fig. 3. Top Left: Illustration of the search task. Initially the upper disk is fixated. The task is to identify the spot with the same color and to perform a saccade to it. Bottom Left: Illustration of a trace task. The target spot is identified by a spatially connected route to the fixation spot. This needs to be traced mentally to select the target. Top Right: Steering a search task requires two essential operations: A readout operation and a spatial selection operation. Selectively focusing the initially fixated spot enhances activity in the color representation with activation red at the focus position. The resulting stronger excitation of the neuron representing the color red in the "Global Color" categories then leads to the spatial selection operation through modulation of all neurons that were activated with the color feature red, while neurons representing green items receive only weak modulatory feedback. Bottom Right: Depiction of the iconization used for defining the network structure. Each box represents a group of neurons connected to other neurons in the way indicated by the connection type.

A simple network that solves the search task along with numerical simulation results of the neuronal activities is presented in Fig. 4. The neurons in the "Spatial Focus" map (indicated by self-excitation arrows in Fig. (4) as part of a working memory maintain activity at the fixation spot until the task is completed. Planning of the next saccade is achieved by the competition between ramping neurons in the "Spatial Saliency" map (c.f. sect. 3). The enhanced activity in the "Color" map at the target spot can now enhance the corresponding neuron in the "Spatial Saliency" map to win the competition (spatial selection part). To prevent the neurons at the fixation spot to win the competition, the "Spatial Saliency" map is additionally inhibited by an inhibition-of-return (IOR) mechanism.

\section{Cascaded Decisions and Early Cortical Areas' Role}

We will now demonstrate how several model stages interact in the network introduced in Fig. [4 to solve a multi-step decision task. For simplicity, we focus our investigation on the FF-FB interaction and will use a hard-coded control mechanism (see [4] for a biological plausible implementation). Such control processes exclusively interact with higher level areas in our model. By using case studies of multiple cascaded tasks, we will demonstrate how early feature maps involved in sensory input processing contribute to the resolution of uncertainty in the decision process. In addition, we show how decisions that were made at higher stages bind their activations via feedback to the distributed representations in 


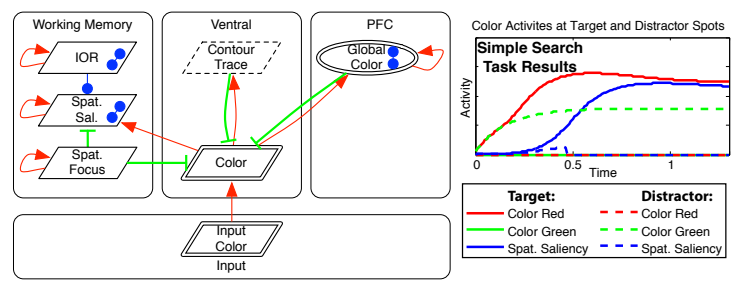

Fig. 4. Left: Sketch of interactions to solve the search task (the map "Contour Trace" is only relevant later). See sect. 4 for a detailed explanation of the processes. Symbols as in Fig. 3 (bottom right). Top Right: Activity in the "Color" and "Spatial Saliency" maps at the target (solid lines) and distractor spot (dashed lines). The global color category is selected by an increased activation level after mutual feature competition between the "Global Color" categories (activity for color red at the target spot dominates). The next fixation location is selected in the "Spatial Saliency" map to relocate the spatial focus and to inhibit the previous fixation location [17] (blue lines).

earlier feature maps which act as neural blackboards. We demonstrate that the response timings and activity modulations in sensory responses resemble similar behavior as reported in physiological investigations.

\subsection{Search and Trace Task}

We choose the search \& trace task (Fig. 5) to compare our results to the results reported by [1, 2]. In comparison with the search task discussed in sect. 4. this new task requires another spatial sub-task (trace task, Fig. 3, left) to be solved before the saccade landing point is finally determined (based on the selection of the same-color spot). The schedule for solving this new composite task consists of two essential steps:

1. Search for "T1" (identified by the same color as the initially fixated disk).

2. Tracing of the line connected to "T1" that separates target "T2" from distractor "D2" (and "D1").

The first step can be solved by our model in the way described in sect. 4. In the second step we employ a diffusive tracing operator (described in sect. 2) to

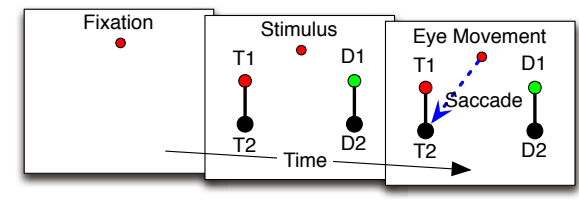

Fig. 5. Search \& trace task [2]. Step 1: Upper disk must be fixated by the test subject. Step 2: Stimulus is shown after upper disk is fixated. A selection between target/distractor is indicated by a saccade to Target 2 "T2". The target is identified by the line to "T1" which has the same color as the fixation spot. Step 3: Saccade in a correct trial (dashed blue arrow). 
facilitate the neural modulation supporting the selection of the target spot "T2" (as suggested by [8]).

\subsection{Sequential Control Using Productions}

Several theories have been proposed for describing the control of cognitive tasks. We employ the basic concept of ACT-R [18] utilizing a simple production system that has been previously learned. Mid- and higher-level model areas (but not the sensory and feature maps and the associated model areas) serve as buffers to carry intermediate activations. The production system watches the model activities of neurons in inter-area competition to win the competition. When a neuron wins the competition a production is fired according to a previously learned plan. The production uses the information of the winning neuron to modify higher model areas and to initiate the next decision process of the network model. Whenever such a production is fired two mechanisms are initiated:

- The task-specific part of a production enhances/inhibits neural activities according to the current goal (this has been learned previously).

- The task unspecific mechanisms slightly inhibit previously winning neurons to favor the selection of other more task relevant neurons in the next decision step (inhibition-of-return mechanism).

We emphasize here that the employment of the production system is intended to simplify the overall model complexity by explicitly focusing on the neural dynamics of sub-task processing and the interaction between parallel feature processing and sequentialized cognitive processes. In the future, we plan to replace the productions by dynamic neural mechanisms.

\subsection{Sequential Productions to Solve a Multi-step Decision Task}

We will now describe the (already learned) productions used to solve the search $\&$ trace task (described above) and show the results of the neuronal dynamics.

- First production (search task described in sect. (4): Set the spatial focus to the fixation spot (maintained by working memory cells). This selectively enhances activity of feedforward activated neurons in the "Color" map (coding one neuron per position and color) such that neurons at the fixation spot get highly activated. Thus, the activity for the color at the fixation spot represents the dominating input for neurons in the global color-category representation (coding one neuron per color). There, neurons are of the ramping type and compete against alternative feature representations. The neuron that wins the competition enhances all similar colors by modulating sensory color activities. Through the enhancement the accumulated activity in the "Spatial Saliency" map targets the next attended position.

- Second production: After the neuron at position "T1" has won the competition between all ramping neurons in the "Spatial Saliency" map, activity in the "Contour Trace" map representation is set at the winning location and the spatial focus is reset. 
- In area "Contour Trace", the activity spreads along the line (that connects the fixation spot with the target position) by the action of the tracing operator that is driven by the input from the perceptual "Color" map. In turn, this enhances activities in the "Color" map via feedback which support ramping neurons in the "Spatial Saliency" map at position "T2" to win their competition. This position is selected as target for eliciting a saccade (the saccade itself as a motor response is not explicitly modeled).
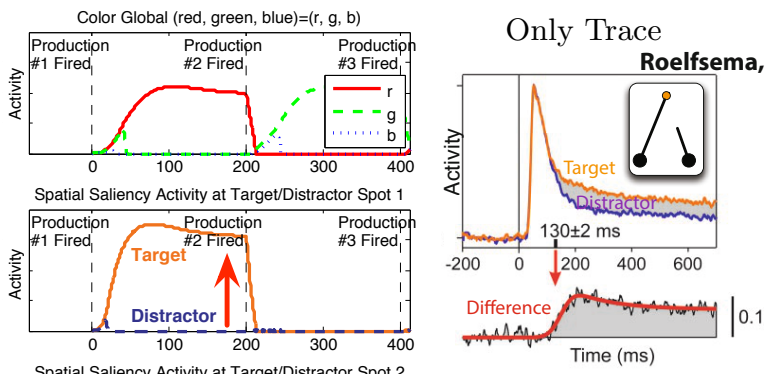

Search \& Trace
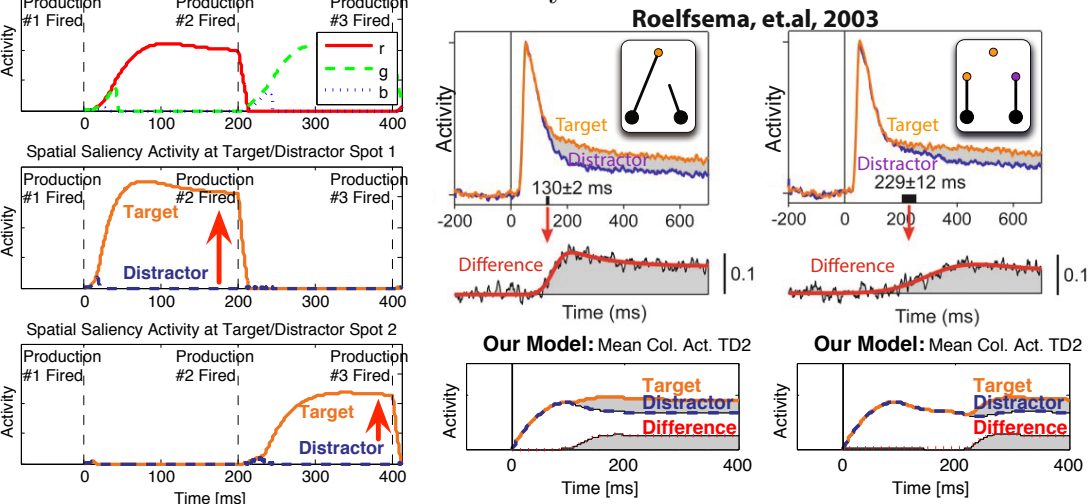

Fig. 6. Left: Temporal dynamics in the search \& trace task. Vertical lines indicate that a production was fired using information indicated by the red arrow. Initially, the spatial focus at the fixation spot enhances the target color red. Top Left: This makes the global category neuron for red winning the competition. Center Left: The modulating enhancement of red in the sensory representation results in enhanced activity at the spot "T1" which causes the feature neuron in the "Spatial Saliency" map at "T1" win the competition. Bottom Left: Second production is fired and triggers the tracing of the curve, which, in turn, leads to a winning competition of neurons at "T2" in the "Spatial Saliency" map. Top Right (four graphs): Neural recordings in a simple trace task (fixation spot connected to target by a line) and in the search \& trace task (reprinted from [2]; (C) 2003 National Academy of Sciences, U.S.A.). Bottom Right: Response phases of the activities of our early sensory model feature map "Color" have a good match with the response phases in the recordings (top).

The temporal dynamics of the relevant neurons at different model stages are shown in Fig. 6. We focus here on how modulatory feedback from global color selection and trace representation leads to delayed response modulations in early sensory feature maps. The results show quantitatively similar response characteristics as for neurons recorded in area V1 [2]. The results support evidence that feedback from higher-level decision related stages coordinates activity representations at the sensory and feature level to generate coherent parallel-serial processing in distributed neural cortical networks 11, 19. 


\section{Discussion and Conclusion}

We propose a neural model architecture that aims to explain how single tasks and the chaining of multiple sub-tasks into composite tasks can be accomplished. In this work, we make three main contributions: (i) We demonstrate how the sub-tasks can be solved by the execution of modular network processes based on interacting neuronal maps located in several distributed model areas. Furthermore, we unify the concepts of serial routing mechanisms and the concept of cortical blackboard representations [11, 9]. (ii) These neural interactions cause an interface problem between serial iterative mechanisms and parallel sensory driven spatial and feature processing. We demonstrate here, how such an interaction that is based on FF and modulating FB interaction can solve the underlying synchronization of responses. We claim that this provides a possible implementation of the sub-task compositions recently proposed in [10] by using a neural model based on gradual activation dynamics of neural firing rate encodings. (iii) The realization of the model architecture we propose, demonstrates how variants of a generic neuron model can be utilized to accomplish the required response properties and, thus, offer a modularization to define neural mechanisms and to build models.

We demonstrate how a sequential control process can interact with higher model areas which in turn indirectly utilize lower model areas via feedback connections. The good quantitative match with electrophysiological recordings provides further evidence that early areas not only receive feedback to enhance response properties but play an essential role in individual decision processes. The model predicts that without feedback interaction, such decision tasks would be much harder to solve or remain even unsolvable. The serialization in which the parallel processing power of early areas is integrated into the decision process is consistent with findings that increased multitasking performance results from higher processing speed [20]. At the moment, we employ a production system to initialize the neural processes related to the individual sub-tasks. This hybrid approach was employed here to simplify the mechanisms that are necessary to initiate processes and representations at the interface, which is an important investigation in neural terms. One of our future investigation will focus on how such sequencing mechanisms can be learned in a neural architecture based on reinforcement principles. Also, concerning further cascaded decision tasks, we will investigate sub-task scheduling and selection over multiple stages. Here, we will study the influences of feature and position information which influences the further selection steps at intermediate stages. The findings will generate predictions that can be tested in further experiments.

Acknowledgments. Research supported by graduate school of Mathematical Analysis of Evolution, Information and Complexity, Ulm University and DFG SFB/TRR 62 . The constructive criticism of three reviewers is gratefully acknowledged. 


\section{References}

1. Moro, S.I., Tolboom, M., Khayat, P.S., Roelfsema, P.R.: Neuronal Activity in the Visual Cortex Reveals the Temporal Order of Cognitive Operations. J. Neurosci. 30(48), 16293-16303 (2010)

2. Roelfsema, P.R., Khayat, P.S., Spekreijse, H.: Subtask Sequencing in the Primary Visual Cortex. PNAS 100(9), 5467-5472 (2003)

3. Ullman, S.: Visual Routines. Cogn. 18(1-3), 97-159 (1984)

4. O'Reilly, R.C., Frank, M.J.: Making Working Memory Work: A Computational Model of Learning in the Prefrontal Cortex and Basal Ganglia. Neural Comput. 18(2), 283-328 (2006)

5. Brosch, T., Neumann, H.: The Combination of HMAX and HOGs in an Attention Guided Framework for Object Localization. In: ICPRAM, vol. 2, pp. 281-288 (2012)

6. Mutch, J., Knoblich, U., Poggio, T.: CNS: a GPU-based Framework for Simulating Cortically-organized Networks. Tech. rep., MIT, Cambridge, MA, USA (2010)

7. Mutch, J., Lowe, D.G.: Object Class Recognition and Localization using sparse Features with limited Receptive Fields. IJCV 80(1), 45-57 (2008)

8. Roelfsema, P.R.: Elemental Operations in Vision. TiCS 9(5), 226-233 (2005)

9. Zylberberg, A., Slezak, D.F., Roelfsema, P.R., Dehaene, S., Sigman, M.: The Brain's Router: A Cortical Network Model of Serial Processing in the Primate Brain. PLoS Comput. Biol. 6(4), 1-23 (2010)

10. Zylberberg, A., Dehaene, S., Roelfsema, P.R., Sigman, M.: The Human Turing Machine: A Neural Framework for Mental Programs. TiCS 15(7), 293-300 (2011)

11. van der Velde, F., de Kamps, M.: Neural Blackboard Architectures of Combinatorial Structures in Cognition. Behav. Brain Sci. 29(1), 37-108 (2006)

12. Bouecke, J.D., Tlapale, E., Kornprobst, P., Neumann, H.: Neural Mechanisms of Motion Detection, Integration, and Segregation: From Biology to Artificial Image Processing Systems. EURASIP JASP 2011(6), 1-22 (2011)

13. Carpenter, G.A., Grossberg, S.: Pattern Recognition by Self-Organizing Neural Networks. A Bradford Book (1991)

14. Grossberg, S., Pilly, P.K.: Temporal Dynamics of Decision-Making During Motion Perception in the Visual Cortex. Vision Research 48(12), 1345-1373 (2008)

15. Roitman, J.D., Shadlen, M.N.: Response of Neurons in the Lateral Intraparietal Area During a Combined Visual Discrimination Reaction Time Task. J. Neurosci. 22(21), 9475-9489 (2002)

16. Ungerleider, L.G., Haxby, J.V.: 'What' and 'Where' in the Human Brain. Current Opinion in Neurobiol. 4(2), 157-165 (1994)

17. Hamker, F.H.: The Emergence of Attention by Population-based Inference and its Role in Distributed Processing and Cognitive Control of Vision. Comput. Vision Image Understand. 100, 64-106 (2005)

18. Anderson, J.R., Bothell, D., Byrne, M.D., Douglass, S., Lebiere, C., Qin, Y.: An integrated Theory of the Mind. Psychol. Rev. 111(4), 1036-1060 (2004)

19. Kveraga, K., Ghuman, A.S., Bar, M.: Top-Down Predictions in the Cognitive Brain. Brain Cogn. 65(2), 145-168 (2007)

20. Dux, P.E., Tombu, M.N., Harrison, S., Rogers, B.P., Tong, F., Marois, R.: Training Improves Multitasking Performance by Increasing the Speed of Information Processing in Human Prefrontal Cortex. Neuron 63(1), 127-138 (2009) 